\title{
Scandinavian Journal of Trauma, Resuscitation and Emergency Medicine reviewer acknowledgement 2015
}

Kristi G. Bache

\section{Contributing reviewers}

The editors of Scandinavian Journal of Trauma, Resuscitation and Emergency Medicine would like to thank all our reviewers who have contributed to the journal in volume 23 (2015).

\section{Muhammad Adrish}

United States of America

Pål Ager-Wick

Norway

Juan Asensio

United States of America

Pongsakorn Atiksawedparit

Thailand

Graham Barker

United Kingdom

Joost Bierens

Netherlands

Paolo Boffano

Italy

Katarina Bohm

Sweden

Mikkel Brabrand

Denmark

Kolbjørn S Brønnick

Norway

Andrzej Cacko

Poland

Muazez Cevik

Turkey

\section{Kirsty Challen}

United Kingdom

Nipon Chattipakorn

Thailand

Jitender Chaturvedi

India

Ivy Cheng

Canada

Damian Clarke

South Africa

Tim Coats

United Kingdom

Maria J Colomina

Spain

Luiz Guilherme Costa

Brazil

Charles Court-Brown

United Kingdom

David Dries

United States of America

Francois-Xavier Duchateau

France

Martin Dworschak

Austria

\section{Evert Eriksson \\ United States of America}

Espen Fevang

Norway

Gary Ford

United Kingdom

Spiros Frangos

Afghanistan

Margaret Fry

Australia

Samuel Galvagno

United States of America

\section{Alexandre Ghuysen}

Belgium

\section{George Giannopoulos}

Greece

David Griffiths

United Kingdom

Carl Gwinnutt

United Kingdom

Samir H Haddad

Saudi Arabia

Kristiann Heesch

Australia

Correspondence: editorial@sjtrem.com

Norwegian Air Ambulance Foundation, Drøbak, Norway 
Johan Herlitz

Sweden

Lars Jacobsen

Norway

Hwan Jun Jae

Korea, South

Marcin Jaracz

Poland

Iosifina Karmaniolou

Greece

Jeffry Kashuk

United States of America

Marius Keel

Switzerland

Kyuseok Kim

Korea, Republic Of

Firas Kobeissy

Lebanon

Antti Koivusalo

Finland

Rudolph Koster

Netherlands

Jinn-Rung Kuo

Taiwan

Jouni Kurola

Finland

Lionel Lamhaut

France

Mark Lehnert

Germany

Ari Leppaniemi

Finland

Chun Lim

United States of America

Gitte Linderoth

Denmark

Anne Lippert

Denmark

Andrew Lockey

United Kingdom

Sigbjørn Løes

Norway
Trine Madsen

Denmark

Ronald Maier

United States of America

Ajai Malhotra

United States of America

Aidan Marsh

United Kingdom

Tara McMorrow

Ireland

Carl Mcqueen

United Kingdom

Jane McVicar

United Kingdom

Terje Meling

Norway

Bruno Meloni

Australia

Pavel Michalek

Czech Republic

Timothy Miller

United States of America

Biswadev Mitra

Australia

Patrick Murphy

United Kingdom

Yih Yng NG

Singapore

Alexander Olaussen

Australia

Peter Paal

Austria

Cameron Palmer

Australia

Michael Pasquale

United States of America

Tommaso Pellis

Italy

Thomas Rea

United States of America

Kristina Rehm

United States of America
Marius Rehn

Norway

Giuseppe Ristagno

Italy

Lindsetmo Rolv-Ole

United States of America

Kurt Ruetzler

United States Minor Outlying Islands

John Santamaria

Australia

Danielle Sartorius

Switzerland

Thomas Scalea

United States of America

Thomas Andersen Schmidt

Denmark

Herbert Schöchl

Austria

David Skinner

South Africa

David Smekal

Sweden

Sanju Sobnach

South Africa

Stephen Sollid

Norway

Kjetil Soreide

Norway

Eldar Soreide

Norway

Philip Stahel

United Kingdom

Giacomo Strapazzon

Italy

Manuel Florian Struck

Germany

Jose Suarez

United States of America

Geir Arne Sunde

Norway

Srinivas Susarla

United States of America 
Gill Sviri

Israel

Hamid R. Talari

Iran, Islamic Republic Of

Julian Wei Tze Tang

United Kingdom

Colman Taylor

Australia

Brian Telesz

United States of America

Peter Teschendorf

Germany

Götz Thomalla

Germany
Tinne Tranberg

Denmark

Javier Urbano

Spain

Charles Wade

United States of America

Andrew Weatherall

Australia

Volker Wenzel

Austria

Jinliang Xing

China

Harlokesh Yadav

India
Junwei Yang

China

Hideo Yasunaga

Japan

Moriwaki Yoshihiro

Japan

Shi-Min Yuan

China

Erik Zakariassen

Norway 